\title{
Application of Rehabilitation Therapy in Elderly Patients with Alzheimer's Disease
}

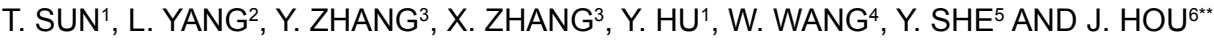 \\ ${ }^{1}$ Department of Neurology, Hainan Hospital of PLA General Hospital, 80th Jianglin Road, Haitang District, Sanya, Hainan \\ Province, 572013, 'Department of Nursing, International Ward in the 2nd medical center of PLA General Hospital, 28th \\ Fuxing Road, Haidian district, Beijing, 100853, ${ }^{3}$ Department of Neurology, The 1st Medical Center of PLA General Hospital, \\ 28th Fuxing Road, Haidian district, Beijing, $100853,{ }^{4}$ Department of Rheumatology, Hainan Hospital of PLA General Hospital, \\ 80th Jianglin Road, Haitang District, Sanya, Hainan Province, 572013, ${ }^{5}$ Department of Respiration, Hainan Hospital of PLA \\ General Hospital, 80 th Jianglin Road, Haitang District, Sanya, Hainan Province, 572013, 6Department of Nursing, PLA General \\ Hospital, 28th Fuxing Road, Haidian district, Beijing, 100853, China
}

\section{Gao et al.: Effect of Liraglutide on Mouse Pre-osteoblast MC3T3-E1 expression}

In order to study the application of rehabilitation therapy in elderly patients with Alzheimer' $s$ disease, 80 elderly patients with Alzheimer' $s$ disease are selected as the research object. The patients are aged 65-85 years with an average age of $\mathbf{7 4 . 2}$ years old. The patients are randomly divided into two groups, 
40 in each group. One group is treated by scraping, and the other group is treated by oral donepezil hydrochloride tablets. MMSE and ADL scores before and after treatment are used to determine the degree of rehabilitation. It is found that the two groups of treatment methods are effective, and curettage therapy and oral western medicine have no significant difference in the efficacy. The MMSE scores of patients are improved, and the ADL scores are decreased, which indicates that curettage without injury can be used instead of oral western medicine which has side effects on human body. Curettage therapy is a long-term effective rehabilitation treatment for patients, which can slow down the process of Alzheimer's disease and reduce the burden of patients and their families.

Key words: Rehabilitation therapy, elderly patients, Alzheimer's disease

Alzheimer's disease(AD) is a chronic neurodegenerative disease characterized by general dementia such as memory impairment, aphasia, agnosia, executive dysfunction and personality and behavioral changes. At the same time, AD is also known as a typical protein conformation disorder (PCDs). More than 30 kinds of PCDs have been found to have very similar pathogenesis with $\mathrm{AD}$, such as cataract, Parkinson' s disease, Huntington's chorea and so on ${ }^{[1]}$. Alzheimer' s disease (AD) is a common senile disease, which is sudden and difficult to be detected and diagnosed in its early stage. The irreversibility of the disease makes the cognitive ability and daily living ability of the elderly gradually lose, which seriously affects the quality of life of the elderly. With the aggravation of China' s aging problem, the number of Alzheimer' $s$ disease patients is also growing rapidly ${ }^{[2]}$. According to a survey, the prevalence of Alzheimer's disease in China is about $3.5 \%$. It is estimated that by 2030 , the number of Alzheimer's patients over 60 years old in China will be 2.8 times that in $2010^{[3]}$. The social and economic burden caused by senile dementia even exceeds that of cardiovascular and cerebrovascular diseases and most malignant tumors. The pathogenesis of Alzheimer' $s$ disease in modern medicine is still unclear ${ }^{[4]}$. There are some problems such as low efficiency of treatment and more adverse reactions. Therefore, Alzheimer' s disease has always been one of the difficult problems that modern biomedicine is constantly tackling ${ }^{[5]}$. In recent years, the treatment of Western medicine is mostly based on the use of intellectual drugs or drugs to improve cognitive impairment. Although it has certain effects, long-term use will also produce certain toxic and side effects. Traditional Chinese medicine (TCM) treatment based on differentiation of symptoms and signs has increasingly shown its advantages. Acupuncture and moxibustion have also achieved positive effects in the clinical treatment of Alzheimer' $s$ disease ${ }^{[6,7]}$. Many patients are afraid of needling, and fear of receiving acupuncture treatment. Therefore, it is necessary to find a treatment method with overall regulation, stable curative effect, small side effects and easy acceptance by patients to improve the symptoms of Alzheimer's disease ${ }^{[8]}$. Scraping therapy is different from traditional scraping. It is characterized by light scraping without scraping. It pays more attention to gentleness, rapidity, fluency and easy operation. Combining with my tutor' s observation in clinic, it is found that many old people with memory decline will have different wrinkles in front of their ears. By stimulating these wrinkles, the symptoms of memory decline can be improved to a certain extent. Therefore, through the design of clinical scientific research, the anterior auricular meridians are regarded as a stimulating part to observe the curettage therapy' $s$ approach to senile dementia in clinic. In this study, 80 patients with Alzheimer's disease in neurology department of Hainan Hospital of PLA General Hospital from July 2013 to July 2018 are randomly divided into treatment group and control group with 40 cases in each group. The treatment group uses thick scraping plate to scrape the Ear Tragus and head. The patients in the control group are treated with donepezil hydrochloride tablets. The informed consent signed by the patients or their family members was obtained and this study was approved by the medical ethics committee of Hainan Hospital of PLA General Hospital. There are many cognitive deficits in $\mathrm{AD}$ diagnosis, which are as follows. Memory deficit refers to the ability of memory, including the decline of the ability of near and far memory. Aphasia refers to the ability of spoken language, which includes obstacles to understanding other people's language ability and the inability to answer other people's questions in an orderly manner. Those with higher education level have reading and writing disabilities. Computing refers to the obstacles to the ability to calculate and recite numbers. Recognition refers to the deterioration of the ability to recognize spatial location and structure. Judgment refers to the deterioration of the ability to judge and recognize people, objects, time and place. Personality refers to a more isolated disposition, cumbersome and repetitive speech, selfish and narrow-minded, 
stubborn, indifferent or unreasonable pleasure, prone to excitement or anger, etc. There are obvious cognitive deficits leading to social impairment, and related functions are also significantly reduced. Thinking refers to the decline of the ability of abstract thinking, such as the inability to explain the meaning of proverbs, to define things, and to distinguish the similarities and differences between two words. Personality refers to the change of personality characteristics and the lack of moral ethics. Most of them are over 60 years old. Course of disease: The onset and development of the disease are slow and the course is long. Within six mo, memory, calculation, judgment and one of the other five mental activities show significant regression and functional deficit, and the disorder is not caused by delirium, major depression, or mental disorder. Alzheimer's disease can be diagnosed according to age and course of disease. Criteria for severity diagnosis: Learning new things is hampered. In some cases, it is impossible to recall what happened before, and there are obvious impairments in non-verbal parts. Based on objective examination, the related evaluation of the above obstacles is obtained. Classification is made according to the following criteria. Mild: Daily life is affected to a certain extent, but patients can still live independently. Long-term memory has not been significantly affected. Moderate: Severe memory impairment has emerged, and its independent life has begun to be significantly affected, possibly with sphincter dysfunction. Severe: There is severe memory disorder. Patients are unable to live a normal life, and must be helped by others. Through the study of medical history, it is found that the intellect declines obviously, and the thinking ability also declines greatly. According to the following criteria for grading: Mild: The patient's daily life has been affected to a certain extent, but the thinking ability is still normal. Complex tasks are difficult. Moderate: The ability to live independently has been significantly affected, and everything is indifferent. Severe: Patients must be helped by others so as to live. In the process of these obstacles, there is no obstacle to normal consciousness. It can be accompanied by emotional and social communication barriers. It is found in clinical diagnosis that the occurrence of this disorder lasts more than six months. Signs of cortical damage are found. Specific manifestations are loss of recognition and uselessness. CT and MRI results also show significant changes.

Inclusion criteria: Patients meet the diagnostic criteria. According to the needs of clinical research and the characteristics of the disease, the age of the subjects is determined to be over $60 \mathrm{y}$ old. The informed consent is signed to enter the research. Exclusion criteria: Patients who receive other treatment at the same time are excluded. Those with obvious or combined syndrome are excluded from end-stage Alzheimer' s disease. Patients with severe neurological deficits, such as aphasia, are excluded. Severe primary diseases, such as cardiovascular, liver, kidney and hematopoietic system, and psychiatric patients are excluded. After the inclusion of cases, those patients who do not meet the diagnostic criteria for Alzheimer' $s$ disease are excluded from incorrect inclusion. Those who meet the inclusion criteria but are unable to cooperate with clinical treatment are excluded. In the course of clinical observation, patients with poor compliance and affecting the effectiveness and safety evaluation are excluded. Serious adverse events, complications and special physiological changes are excluded, and those who are unsuitable for further clinical treatment are excluded. For various other reasons, patients who do not withdraw from clinical observation before the end of the course of treatment are excluded. The experimental flow chart is shown in fig. 1. Treatment group (scraping therapy): Instrument is thick scraping plate. Treatment site is front of tragus and head, as shown in fig 2. Operational methods: Operation is carried out on Alzheimer' $s$

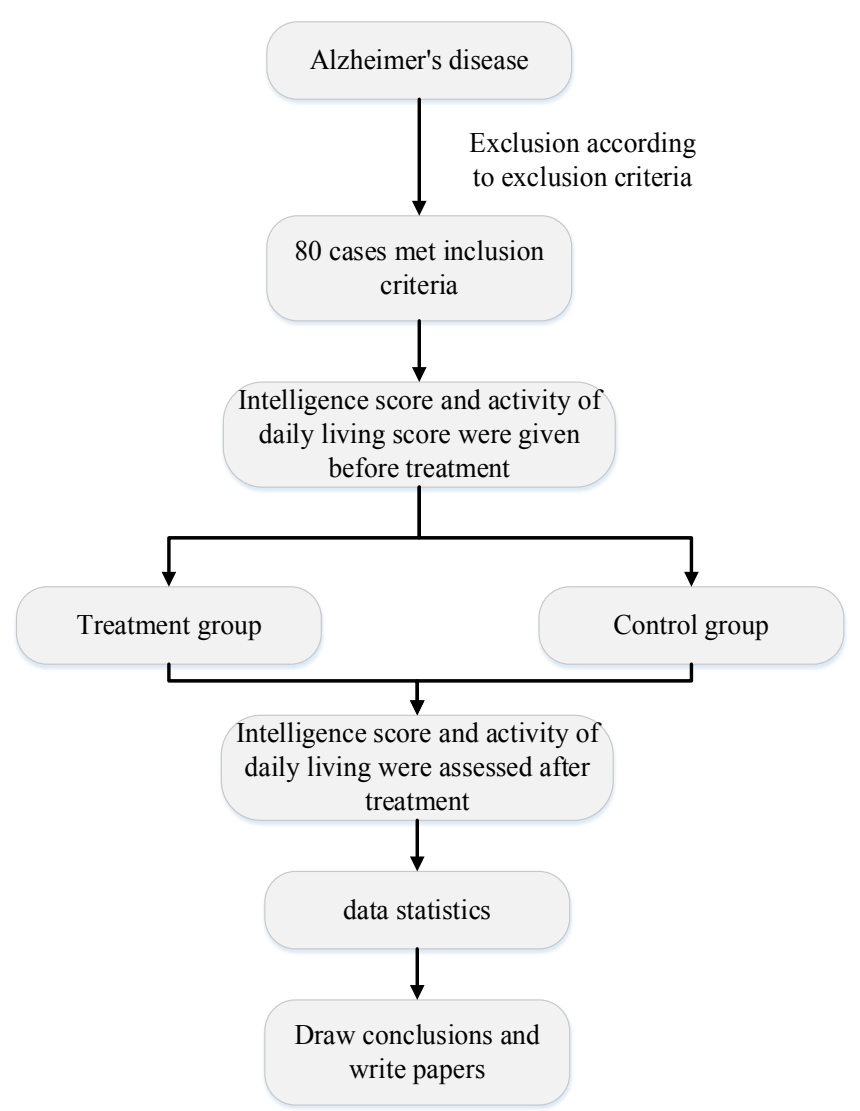

Fig. 1: Experimental flow chart

rmaceutical Sciences 


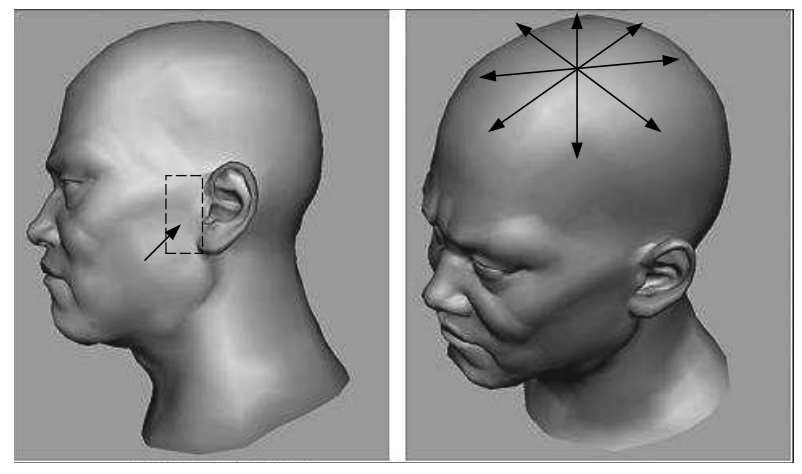

Fig. 2: Scraping site

disease patients with anterior tragus (from the posterior edge of temples to the anterior lobe) from top to bottom, and the manipulation should be gentle, fast and smooth. The intensity should be basically unchanged skin color, fever and no pain. The strength is basically unchanged by the color of the skin. Fever without pain is a criterion. The frequency is 2 times per second, and each side should be scraped 35 times. Head Scraping: The head is scraped in all directions with a slightly heavy force, in a radial manner centered on Baihui. Course of treatment: once a day, six times a week, two weeks as a course of treatment, two consecutive months, a total of four courses.

The control group (oral western medicine group): $5 \mathrm{mg}$ donepezil hydrochloride tablets are taken orally once a day before bedtime. Two weeks is a course of treatment, two months in a row, a total of four courses of treatment. Simple Mental State Assessment Scale (MMSE): Questions and records are conducted by the evaluator, including 10 points of orientation, 3 points of memory, 3 points of recall, 9 points of language ability, 5 points of attention and calculation, with a full score of 30 points, of which 27-30 points are normal and score $<27$ points are cognitive impairment. Activity of daily living (ADL) scale: Through the evaluation and recording of the problem, 1 point $=$ patients can do; 2 points $=$ some difficulties, but patients can complete; 3 points $=$ need help; 4 points $=$ cannot do at all. When the patient has never done it, but is competent, the score is 1 . When the patient has never done it, but there are difficulties to do it without the help of others, the score is 2 . When the patient has never done it, but the patient can do it with the help of others, the score is 3 . When the patient has never done it and cannot do it, the score is 4 . The scoring range is $20-80$, and the score of $>23$ is cognitive impairment. The criteria for judging the efficacy of new Chinese medicines in the treatment of dementia are determined according to the Guiding Principles for Clinical Research of New Chinese
Medicines (2002) formulated by the Ministry of Health. Clinical control: The main symptoms basically return to normal and the orientation is sound. Patients can correctly answer questions, take care of themselves and resume general social activities. Markedly effective: Most of the main symptoms return to normal, and the orientation is basically sound. Patients can correctly answer questions, and take care of themselves. The reaction is general. Effective: The main psychiatric symptoms are alleviated or partially disappeared. The patient' $s$ daily life is basically self-care and they can answer questions correctly. However, reaction is still slow and the intelligence and personality are still impeded. Invalidity: The main symptoms do not improve, and it continues to develop.

SPSSA18.0 statistical software is used to analyze the clinical data that meet the inclusion criteria. Gender is tested by $\mathrm{X}^{2}$ test, age, MMSE score and ADL score are tested by $\mathrm{t}$ test, and overall efficacy is tested by rank sum test. Significance t-test is used for measuring data in accordance with normal distribution, which is expressed by $\mathrm{x} \pm \mathrm{s}$. The test significance level is set to 0.05 . P $>0.05$, there is no statistical difference. If $\mathrm{P}<0.05$, there is statistical difference. In this study, a total of 80 subjects are selected, each group of 40 patients with Alzheimer' $\mathrm{s}$ disease successfully completed the treatment. Among them, 43 are males $(53.75 \%)$ and 37 are females $(46.25 \%)$. The age of onset is 65-85 years old, with an average age of 75.4 years old. The gender ratio of the two groups is shown in fig. $3 \mathrm{~A}$, and the degree of the disease is compared with fig. 3B. There is no significant difference between the treatment group and the control group in gender and severity of the disease $(\mathrm{P}>0.05)$. There is no statistical significance and comparability between the two groups. The MMSE scores of the patients in the treatment group before treatment, after the first, second, third and fourth courses of treatment, and the MMSE scores of the patients in the control group after the end of treatment are collected. The trend of MMSE score in each course of treatment in the treatment group is shown in fig. 4A. The scores of the two groups before and after treatment are shown in fig. 4B. There is no significant difference in MSE score between the two groups before treatment $(\mathrm{P}>0.05)$, which is comparable. After treatment, the scores of MMSE in both groups are higher than those before treatment, with statistical difference $(\mathrm{P}<0.05)$, indicating that both methods can improve Alzheimer' $s$ disease. After treatment, there is no significant difference in the scores of MMSE between the two groups $(\mathrm{P}>0.05)$, and there is no significant 

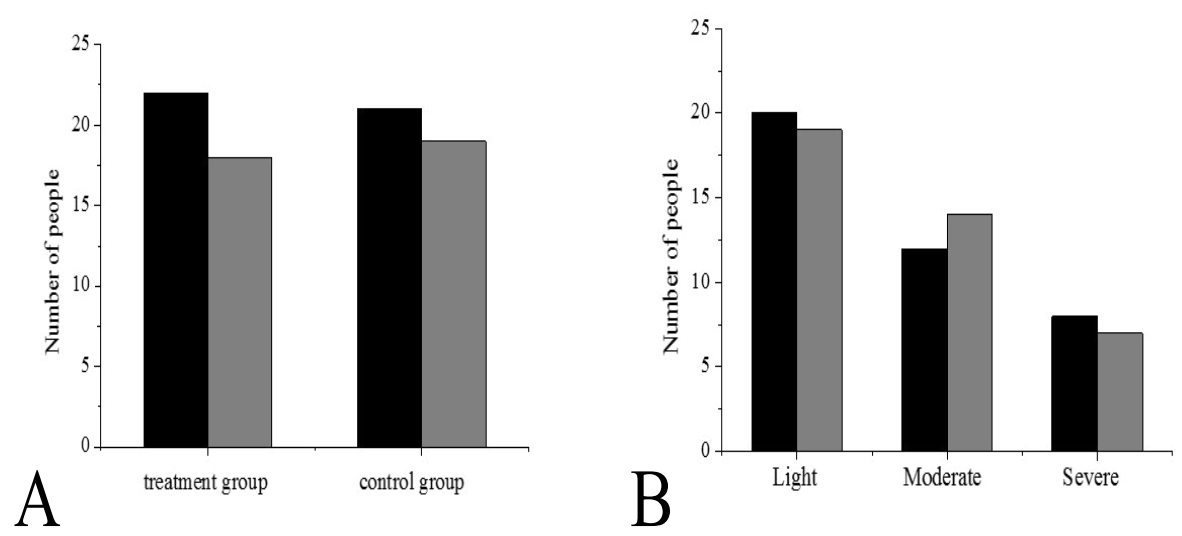

Fig. 3: General situation of alzheimer' s disease (a: gender distribution of two groups b: comparisons of severity of disease between two groups)
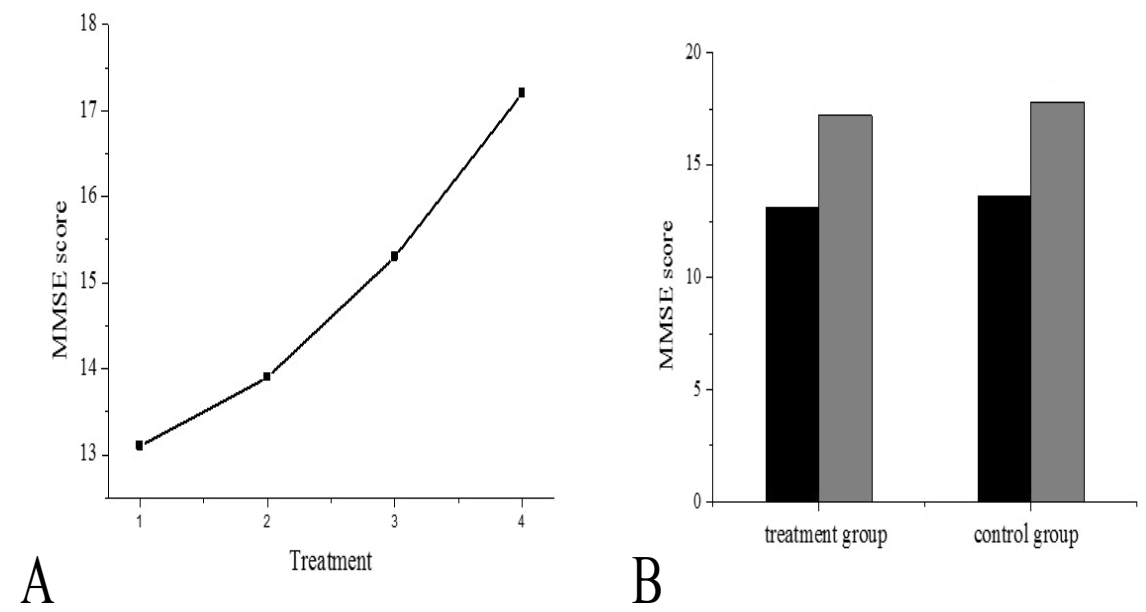

Fig. 4: MMSE score (a: the change trend of patients' scores in each course of treatment; $b$ : the changes of scores before and after treatment in two groups)

difference in the therapeutic effect between the treatment group and the control group. It shows that curettage therapy and oral western medicine have similar effects on improving MMSE score. The ADL scores of the patients in the treatment group before treatment, after the first, second, third and fourth courses of treatment, and the ADL scores of the patients in the control group after the end of treatment are collected. Statistical analysis shows that there is no significant difference in ADL scores between the two groups before treatment $(\mathrm{P}>0.05)$, which is comparable. After treatment, ADL scores of the two groups are decreased compared with those before treatment, with statistical difference $(\mathrm{P}<0.05)$, indicating that both treatment methods can improve Alzheimer's disease. After treatment, there is no significant difference in ADL scores between the two groups $(\mathrm{P}>0.05)$, indicating that curettage and oral western medicine have similar effects on improving ADL scores. Statistical analysis shows that there is no significant difference in the evaluation of curative effect between the two groups after treatment $(\mathrm{P}>0.05)$, and there is no significant difference between the treatment group and the control group, as shown in fig. 6. In 80 patients with Alzheimer' $\mathrm{s}$ disease, the onset age is 65-85 years old, and the average age is 75.4 years old. After comparing curettage and oral western medicine, it is found that the two groups of treatment methods are effective, and curettage and oral western medicine have no significant difference in the efficacy. The MMSE scores of patients are improved, and the ADL scores are decreased, which indicates that curettage without injury can be used instead of oral western medicine which have side effects on human body. Curettage is a long-term effective rehabilitation therapy for patients. Although there is no effective way to cure Alzheimer' s disease, this method can effectively improve the aphasia of most patients, slow down the disease process of Alzheimer' s disease, and reduce the burden of patients and their families. 

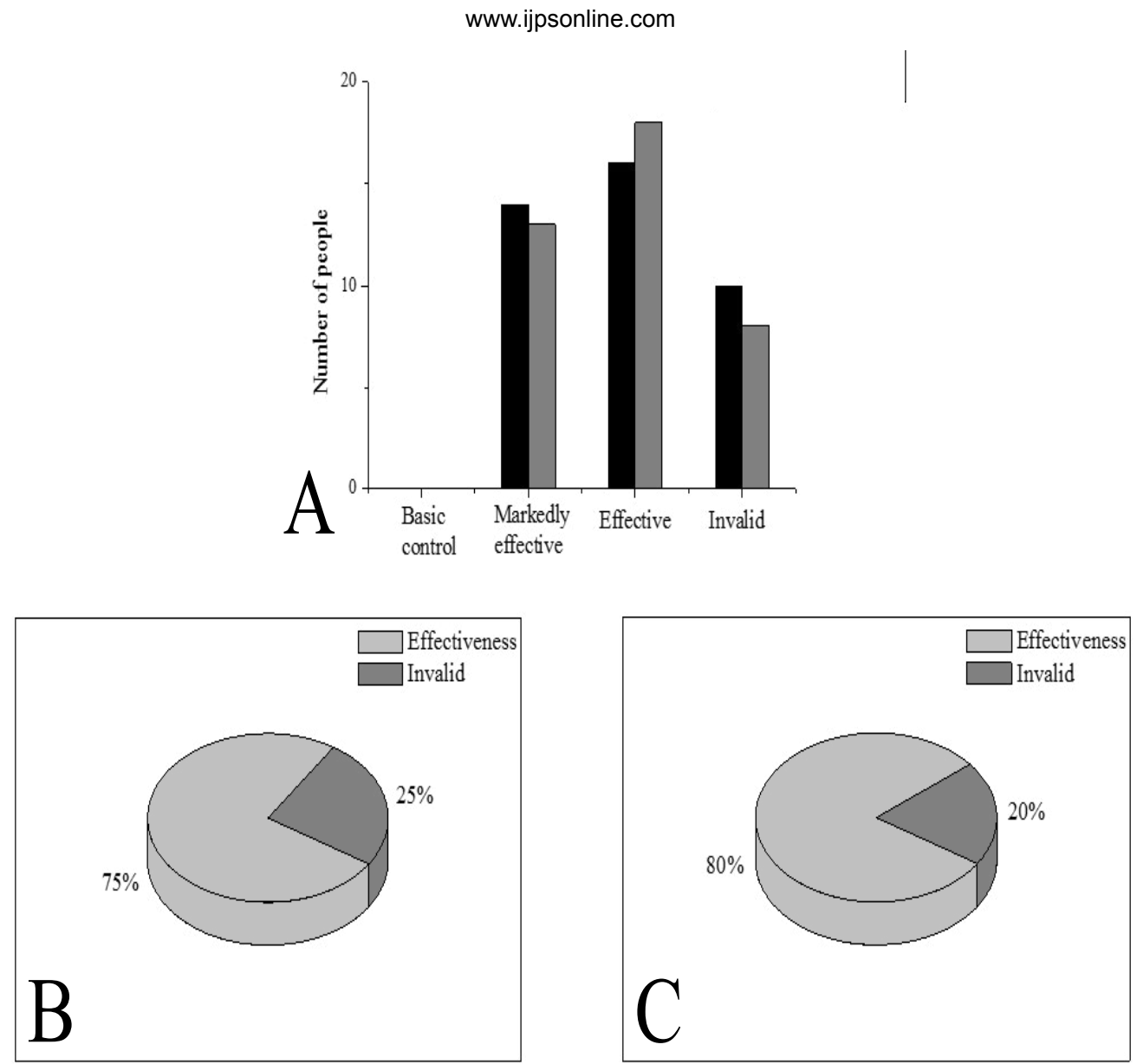

Fig. 5: ADL score (a: the change trend of patients' scores in each course of treatment; b: the changes of scores before and after treatment in two groups)
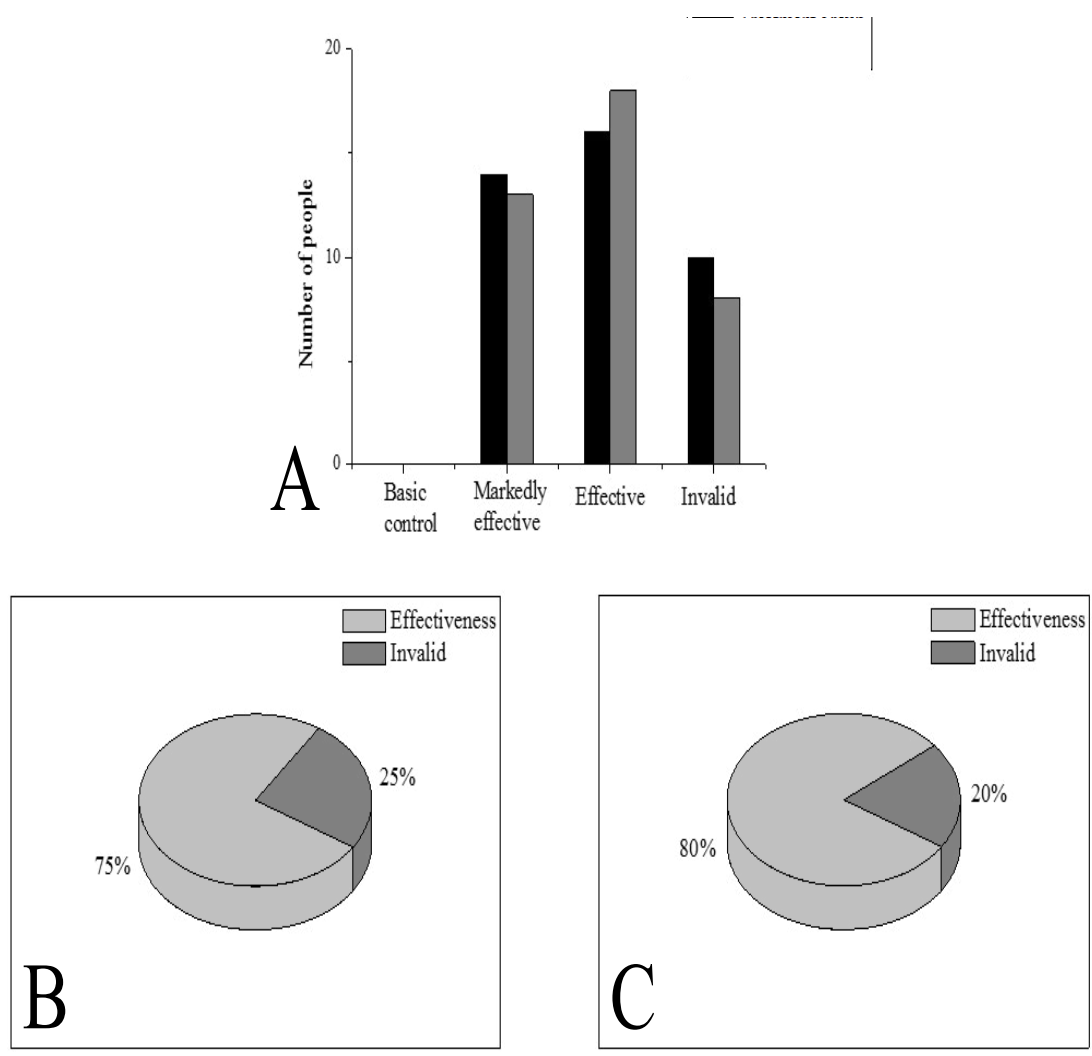

Fig. 6: Evaluation of efficacy (a: patients are basically under control, contrast the number of markedly effective, effective and ineffective people. b: effective rate (\%) of patients in treatment group. c: effectiveness (\%) of the control group) 


\section{Acknowledgment}

This work was supported by Sanya health care scientific innovation project (2018YW18).

\section{REFERENCES}

1. Dumanski JP, Lambert JC, Rasi C. Mosaic loss of chromosome $\mathrm{Y}$ in blood is associated with Alzheimer disease. The American Journal of Human Genetics, 2016; 98(6): 1208-1219.

2. Sweeney MD, Sagare AP, Zlokovic BV. Blood-brain barrier breakdown in Alzheimer disease and other neurodegenerative disorders. Nature Reviews Neurology, 2018; 14(3): 133.

3. Jack CR, Bennett DA, Blennow K. A/T/N: an unbiased descriptive classification scheme for Alzheimer disease biomarkers. Neurology, 2016; 87(5): 539-547.

4. Van Cauwenberghe C, Van Broeckhoven C, Sleegers K. The genetic landscape of Alzheimer disease: clinical implications and perspectives. Genetics in Medicine, 2016; 18(5): pp. 421.

5. Palop JJ, Mucke L. Network abnormalities and interneuron dysfunction in Alzheimer disease. Nature Reviews Neuroscience, 2016; 17(12): 777.
6. Colonna M, Wang Y. TREM2 variants: new keys to decipher Alzheimer disease pathogenesis. Nature Reviews Neuroscience, 2016; 17(4): 201.

7. Silvestre FJ, Lauritano D, Carinci F, Silvestre-Rangil J, Martinez-Herrera M, Del Olmo A. Neuroinflammation, Alzheimer's disease and periodontal disease: is there an association between the two processes? J Biol Regul Homeost Agents, 2017; 31(2 Suppl 1): 189-196.

8. Iosa M, Morone G, Fusco A. Leap motion-controlled videogame-based therapy for rehabilitation of elderly patients with subacute stroke: a feasibility pilot study. Topics in stroke rehabilitation, 2015; 22(4): 306-316.

This is an open access article distributed under the terms of the Creative Commons Attribution-NonCommercial-ShareAlike 3.0 License, which allows others to remix, tweak, and build upon the work non-commercially, as long as the author is credited and the new creations are licensed under the identical terms

This article was originally published in Special issue on "Trends in therapeutic Management of Various Conditions" Indian J Pharm Sci 2020:82(3) spl issue 6;115-121 